\title{
Lyapunov exponents for some isotropic random matrix ensembles
}

\author{
P. J. Forrester ${ }^{1}$ and Jiyuan Zhang ${ }^{2}$ \\ ARC Centre of Excellence for Mathematical and Statistical Frontiers, \\ School of Mathematics and Statistics, The University of Melbourne, Victoria 3010, \\ Australia.
}

\begin{abstract}
A random matrix with rows distributed as a function of their length is said to be isotropic. When these distributions are Gaussian, beta type I, or beta type II, previous work has, from the viewpoint of integral geometry, obtained the explicit form of the distribution of the determinant. We use these result to evaluate the sum of the Lyapunov spectrum of the corresponding random matrix product, and we further give explicit expressions for the largest Lyapunov exponent. Generalisations to the case of complex or quaternion entries are also given. For standard Gaussian matrices $X$, the full Lyapunov spectrum for products of random matrices $I_{N}+\frac{1}{c} \mathrm{X}$ is computed in terms of a generalised hypergeometric function in general, and in terms of a single single integral involving a modified Bessel function for the largest Lyapunov exponent.
\end{abstract}

\section{Introduction}

Let $X$ be a real $N \times N$ random matrix. A basic question is to ask about the distribution of its determinant. Since $|\operatorname{det} X|=\left(\operatorname{det} X^{T} X\right)^{1 / 2}$, the determinant of the positive definite matrix $X^{T} X$ provides a natural extension of this question to the case of $n \times N(n \geq N)$ rectangular matrices. In the latter setting, thinking of $X$ as a multi-dimensional data matrix, with the measured quantities corresponding to the columns and each with mean zero, $X^{T} X$ is up to proportionality the empirical correlation matrix between these quantities. Moreover, $\left(\operatorname{det} X^{T} X\right)^{1 / 2}$ has the geometrical interpretation as the Hausdorff volume of the parallelotope generated by the columns of $X$; see e.g. [21].

Let $\left\{\tau_{j}\right\}_{j=1}^{N}$ denote the singular values of $X$. Since $|\operatorname{det} X|=\prod_{l=1}^{N} \tau_{l}$, one approach to this question is to make use of knowledge of the distribution of the singular values. For example, in the case that $X$ is an $N \times N$ standard

\footnotetext{
${ }^{1}$ pjforr@unimelb.edu.au

jiyuanz@student.unimelb.edu.au
} 
Gaussian matrix, it is known (see e.g. [9, Prop. 3.22 with $\beta=1, n=m=N]$ ) that the joint probability density function (PDF) for the squared singular values $\lambda_{l}=\tau_{l}^{2}$ is proportional to

$$
\prod_{l=1}^{N} \lambda_{l}^{-1 / 2} e^{-\lambda_{l} / 2} \chi_{\lambda_{l}>0} \prod_{1 \leq j<k \leq N}\left|\lambda_{k}-\lambda_{j}\right|
$$

where $\chi_{J}=1$ for $J$ true and $\chi_{J}=0$ otherwise. Studying the distribution of $|\operatorname{det} X|^{2}=\prod_{l=1}^{N} \lambda_{l}$ through its moments then calls for the evaluation of

$$
\int_{0}^{\infty} d \lambda_{1} \cdots \int_{0}^{\infty} d \lambda_{N} \prod_{l=1}^{N} \lambda_{l}^{-1 / 2+s} e^{-\lambda_{l} / 2} \prod_{1 \leq j<k \leq N}\left|\lambda_{k}-\lambda_{j}\right| .
$$

This is a particular (limiting) Selberg integral (see e.g. [9. \$4.7]), and as such can be evaluated as a product of gamma functions to give

$$
\left\langle\prod_{l=1}^{N} \lambda_{l}^{s}\right\rangle_{X}=\prod_{j=1}^{N} \frac{2^{s} \Gamma(s+j / 2)}{\Gamma(j / 2)} .
$$

With $\chi_{j}^{2}$ denoting the chi-squared distribution with $j$ degrees of freedom, we read off from this that

$$
\left\langle\prod_{l=1}^{N} \lambda_{l}^{s}\right\rangle_{X}=\prod_{j=1}^{N}\left\langle\lambda_{j}^{s}\right\rangle_{\chi_{j}^{2}}
$$

and hence (see e.g. [24])

$$
|\operatorname{det} X|^{2} \stackrel{\mathrm{d}}{=} \prod_{j=1}^{N} \chi_{j}^{2}
$$

Our focus in the present paper relates to a different viewpoint on 1.4, which in fact can be traced back to the work of Bartlett [2]. Instead of decomposing $X$ in terms of its singular values, the strategy is to use the $Q R$ (Gram-Schmidt) decomposition in which $Q$ is a real orthogonal matrix consisting of the Gram-Schmidt basis as its columns, and $R=\left[r_{j k}\right]_{j, k=1}^{N}$ is an upper triangular matrix with positive entries on the diagonal. With $(d Y)$ denoting the product of differentials of the matrix $Y$, the change of variables formula (see e.g. [24])

$$
(d Y)=\prod_{l=1}^{N} r_{l l}^{N-l}(d R)\left(Q^{T} d Q\right),
$$

and the facts that $\prod_{j, k=1}^{N} e^{-x_{j k}^{2} / 2}=\prod_{j \leq k}^{N} e^{-r_{j k}^{2} / 2}$, imply that each variable $r_{j j}^{2}$ has distribution $\chi_{N-j+1}^{2}$. Noting that

$$
\operatorname{det} X^{T} X=\prod_{j=1}^{N} r_{j j}^{2}
$$


then reclaims (1.4.

The technique of derivation of (1.4) using the $\mathrm{QR}$ rather than singular value decomposition is a more powerful method, Thus it provides for the exact specification of the distribution of $|\operatorname{det} X|^{2}$ for some ensembles of matrices $X$ with distribution invariant under the transformation

$$
X \mapsto X Q,
$$

for $Q$ a real orthogonal matrix, but otherwise not a function of $X^{T} X$. The latter point means in particular that such ensembles do not permit a formula analogous to 1.1 for the PDF of the squared singular values. An example is the so called uniform Gram ensemble, in which each row of $X$ is chosen uniformly from the unit $(N-1)$-sphere. Use of the $Q R$ decomposition gives that [30, 33]

$$
|\operatorname{det} X|^{2} \stackrel{\mathrm{d}}{=} \prod_{l=1}^{N} \operatorname{Beta}[(N-l+1) / 2,(l-1) / 2],
$$

where Beta $[\alpha, \beta]$ denotes the beta distribution with parameters $(\alpha, \beta)$.

In Section 2 of this paper results on volumes of random parallelotopes in the integral geometry literature will be used to specify random matrix ensembles with the invariance 1.7 which permit results analagous to 1.4 and 1.8 . The entries of each row of $X$ are drawn from a Gaussian, beta type I, or beta type II distribution dependent only on the length of the row. We show that these results can be extended to the case that the matrices in the ensembles have complex, or quaternion, entries rather than being real valued as in the integral geometry setting. There is an application of these results to computations relating to the Lyapunov spectrum $\left\{\mu_{j}\right\}_{j=1}^{N}$ for the random product matrix

$$
P_{m}=X_{m} X_{m-1} \cdots X_{1}
$$

where each $X_{i}$ is chosen independently from an ensemble with the invariance (1.7). The main point underlying this is that the formula of Oseledec [26] and Raghunathan [29] for the sum of the first $k$ largest exponents

$$
\mu_{1}+\cdots+\mu_{k}=\sup \lim _{m \rightarrow \infty} \frac{1}{m} \log \operatorname{Vol}_{k}\left\{y_{1}(m), \ldots, y_{k}(m)\right\} \quad(k=1, \ldots, d),
$$

where $y_{j}(m):=P_{m} y_{j}(0)$, the sup operation is over all sets of linearly independent vectors $\left\{y_{1}(0), \ldots, y_{k}(0)\right\}$ and $\operatorname{Vol}_{k}$ refers to the volume of the parallelotope generated by the given set of $k$ vectors, simplifies in the setting of the invariance [1.7]. Thus, then [4, 25]

$$
\mu_{1}+\cdots+\mu_{k}=\left\langle\log \operatorname{det}\left(X_{N \times k}^{T} X_{N \times k}\right)^{1 / 2}\right\rangle,
$$


where $X_{N \times k}$ is the restriction of $X$ to its first $k$ columns. The results of Section 2 apply directly to the case $k=N$ of 1.11 . An analogous study can be carried out for the products of independent random matrices of the form

$$
I_{N}+\frac{1}{c} X
$$

where $X$ is a standard Gaussian matrix. Although the resulting expression is computationally more difficult, there is simplification in the limit $c \rightarrow \infty$.

In Section 3 we address the problem of using 1 1.12 to compute the largest Lyapunov exponent $\mu_{1}$ for the ensembles of Section 2, and give evaluations in terms of a single integral. It is well known that for products of standard Gaussian random matrices the RHS of [1.10 can be computed explicitly [25]. We show in Section 4 that for product ensembles based on 1.12, an evaluation of (1.10) in terms of a generalised hypergeometric function can be given. For the largest Lyapunov exponent, a much simpler expression, involving only a single integral of standard special functions, is also obtained.

\section{Distribution of the determinant for some invari- ant ensembles}

\subsection{Isotropic rows}

Let the row vector $\mathbf{x}=\left(x_{1}, \ldots, x_{N}\right)$ be sampled from a distribution with one of the PDFs

$$
\begin{cases}\left(2 \pi \sigma^{2}\right)^{-N / 2} e^{-|\mathbf{x}|^{2} / 2 \sigma^{2}}, \mathbf{x} \in \mathbb{R}^{N} & \text { (Gaussian) } \\ \pi^{-N / 2}(\nu / 2)_{N / 2}\left(1-|\mathbf{x}|^{2}\right)^{v / 2-1}, \mathbf{x} \in B_{N} & \text { (Beta type I) } \\ \pi^{-N / 2}(\omega / 2)_{N / 2}\left(1+|\mathbf{x}|^{2}\right)^{-(N+\omega) / 2}, \mathbf{x} \in \mathbb{R}^{N} & \text { (Beta type II). }\end{cases}
$$

Here $(c)_{n}=\Gamma(c+n) / \Gamma(c)$ and $B_{N}$ denotes the unit ball in $\mathbb{R}^{N}$. We require $\sigma>0$ in the Gaussian weight, $v>0$ in the beta type I weight (the limit $v \rightarrow 0^{+}$corresponds to the uniform Gram ensemble) and $\omega \geq 0$ in the beta type II weight. Being functions of $|\mathbf{x}|^{2}$, each of these has the vector invariance corresponding to $[1.7, \mathbf{x} \mapsto \mathbf{x} R$. In the terminology of [31] the distribution is then referred to as isotropic.

The Gaussian case is realised by choosing each $x_{i}$ in $\mathbf{x}$ to be an independent normal random variable with standard deviation $\sigma$. For $v \in \mathbb{N}_{0}$, the distribution corresponding to the beta type I weight can be sampled by choosing a vector uniformly at random on the unit sphere in $\mathbb{R}^{N+v}$, then restricting 
to the first $N$ components; this follows from e.g. [9. Eq. (3.113)]. For $\omega>0$, the distribution corresponding to the beta type II weight can be sampled by choosing $w \stackrel{\mathrm{d}}{=} \chi_{\omega}^{2}$, and then forming $(1 / \sqrt{w}) \mathbf{v}$ where $\mathbf{v}$ is a $1 \times N$ row vector of standard Gaussians [31].

We form three random matrix ensembles by choosing rows independently according to distributions corresponding to the three weights in 2.1), allowing for the parameters $\sigma, v$ and $\omega$ to be different in the different rows. We remark that in the Gaussian case the random matrix can equivalently be specified as the product

$$
\Sigma^{1 / 2} G
$$

where $\Sigma$ is the diagonal matrix $\operatorname{diag}\left[\sigma_{1}^{2}, \ldots, \sigma_{N}^{2}\right]$, and $G$ has independently distributed standard Gaussian elements. Results in [31] give the distribution of the determinant and its moments. In addition to the $\chi^{2}$ and beta distributions, also required is the beta prime distribution, to be denoted BetaP[a,b], which is supported on $(0, \infty)$ and has density proportional to $y^{a-1} /(1+y)^{a+b}$ (see [14] for another recent application of the latter in random matrix theory).

Proposition 1. (Ruben) For the Gaussian weight [31. Eqs. (29), (28)]

$$
\left\langle|\operatorname{det} X|^{2 \alpha}\right\rangle=\prod_{l=1}^{N}\left(2 \sigma_{l}^{2}\right)^{\alpha}((N-l+1) / 2)_{\alpha}
$$

and

$$
|\operatorname{det} X|^{2} \stackrel{\mathrm{d}}{=} \prod_{l=1}^{N} \sigma_{l}^{2} \chi_{l}^{2}
$$

(cf. 2.1] and [1.4); for the beta type I weight [31. Eqs. (36), (35)]

$$
\left\langle|\operatorname{det} X|^{2 \alpha}\right\rangle=\prod_{l=1}^{N} \frac{((N-l+1) / 2)_{\alpha}}{\left(\left(N+v_{l}\right) / 2\right)_{\alpha}}
$$

and

$$
|\operatorname{det} X|^{2} \stackrel{\mathrm{d}}{=} \prod_{l=1}^{N} \operatorname{Beta}\left[(N-l+1) / 2,\left(v_{l}+l-1\right) / 2\right] ;
$$

for the beta type II weight [31. Eqs. (34), (33)]

$$
\left\langle|\operatorname{det} X|^{2 \alpha}\right\rangle=\prod_{l=1}^{N}((N-l+1) / 2)_{\alpha}\left(\omega_{l} / 2\right)_{-\alpha}
$$

and

$$
|\operatorname{det} X|^{2} \stackrel{\mathrm{d}}{=} \prod_{l=1}^{N} \operatorname{BetaP}\left[(N-l+1) / 2, \omega_{l} / 2\right]
$$


Note that the case $v_{l}=0(l=1, \ldots, N)$ of 2.5 reclaims (1.11). Also, we remark that random polytopes formed by the convex hull of the columns of $X^{T}$, in the case that $X$ is of size $n \times N(n>N)$ with rows chosen according to one of the distributions (2.1), have been the subject of the recent studies [18, 19].

The method of proof of Proposition 1 implied by working due to Mathai [20] formulated two decades after [31], relies on the QR (Gram-Schmidt) decomposition and the corresponding change of variables formula (1.5). This strategy can be generalised to the case that the matrix $X$ has complex entries, or has quaternion entries, with rows distributed according to one of the three specifications in 2.1. There are two real components in a complex number and four in a quaternion. This requires that $N$ in 2.1) be replaced by $\beta N$, where $\beta=1,2,4$ for real, complex, quaternion entries respectively, and also in the beta type I case that $v$ be replaced by $\beta v$.

In the case of complex entries, $Q$ is complex unitary and will be denoted $U$. The matrix $R=\left[r_{j k}\right]_{j, k=1}^{N}$ is again upper triangular with positive entries on the diagonal, but now the strictly upper triangular entries are complex. The corresponding change of variables formula reads (see e.g. [9. Prop. 3.2.5])

$$
(d X)=\prod_{j=1}^{N} r_{j j}^{2(N-j)+1}(d T)\left(U^{\dagger} d U\right)
$$

and analogous to 1.6

$$
\operatorname{det} X^{\dagger} X=\prod_{j=1}^{N} r_{j j}^{2}
$$

We say a $2 N \times 2 N$ matrix $X$ has quaternion entries if it consists entirely of $2 \times 2$ blocks of the form

$$
\left[\begin{array}{cc}
z & w \\
-\bar{w} & \bar{z}
\end{array}\right]
$$

In the QR (Gram-Schmidt) decomposition the matrix $Q$ is complex unitary with quaternion entries, and is to be denoted by $U$. The matrix $R$ is now block upper triangular, with diagonal entries $r_{j j}\left[\begin{array}{ll}1 & 0 \\ 0 & 1\end{array}\right], r_{j j}>0$, (a positive scalar quaternion) and the strictly upper triangular entries are quaternions. For the change of variables formula we have ([9, Exercises 3.2 q.5(i)], [8, Lemma 2.7]

$$
(d X)=\prod_{j=1}^{N} r_{j j}^{4(N-j)+3}(d R)\left(U^{\dagger} d U\right) .
$$


Note that as a $2 N \times 2 N$ complex matrix, $X$ would satisfy 2.10 with the RHS squared. However, regarding $X$ as a quaternion matrix the appropriate definition (see e.g. [9]. Def. 6.1.2]) gives that 2.10 is again to be used as specifying the determinant.

In terms of the notation $\beta$ introduced in the paragraph above 2.9 , we remark that $1.5,2.9)$ and 2.12 can be summarised by the one expression

$$
(d X)=\prod_{j=1}^{N} r_{j j}^{\beta(N-j+1)-1}(d R)\left(U^{\dagger} d U\right) .
$$

Moreover, upon applying the QR (Gram-Schmidt) decomposition to $X^{\dagger}$ we see that

$$
\left(X X^{\dagger}\right)_{j j}=\left|\mathbf{x}_{j}\right|^{2}=\sum_{l=1}^{j} r_{l j}^{2}
$$

where in the quaternion case the notation $(\cdot)_{j j}$ refers to the scalar multiple of the identity in position $(j j)$ of the diagonal and $\mathbf{x}_{j}$ refers to the $j$-th row of $X$ regarded as a matrix of quaternions. On this latter point note that with $w=u+i v$ and $z=x+i y$ in 2.11 , the modulus squared of the corresponding quaternion is equal to $u^{2}+v^{2}+x^{2}+y^{2}$. This is in keeping with the presentation of a quaternion in terms of its units $\{1, i, j, k\}$ as a linear combination with real components, $u+v i+x j+y k$.

Suppose that row $j$ of the matrix $X$ has a distribution with $\operatorname{PDF} f_{j}\left(\left|\mathbf{x}_{j}\right|^{2}\right)$. Then it follows from 2.13 and 2.14 that the joint PDF of $\left\{r_{j j}\right\}$ is proportional to

$$
\prod_{j=1}^{N} r_{j j}^{\beta(N-j+1)-1} \int_{\left\{r_{j l}\right\}_{l=1}^{j-1}} f_{j}\left(\sum_{l=1}^{j} r_{l j}^{2}\right) \prod_{l=1}^{j-1} d r_{j l} \text {. }
$$

Note that $d r_{j l}=\prod_{s=1}^{\beta} d r_{j l}^{(s)}$, where $d r_{j l}^{(s)}$ are the differentials associated the independent real numbers which make up $r_{j l}$. For the particular $f_{j}$ as implied by $2.1,2.15$ has the property that its dependence on $\left\{r_{j j}\right\}$ factorises in each of the real, complex and quaternion cases. This allows Proposition 1 to be correspondingly generalised.

Proposition 2. Consider the random matrix ensembles with rows distributed according to 2.1), appropriately generalised as noted in the paragraph above (2.9) to allow for complex or quaternion entries. Use the notation $\beta$ of that paragraph to distinguish the number field. For the Gaussian weight

$$
\left\langle|\operatorname{det} X|^{2 \alpha}\right\rangle=\prod_{l=1}^{N}\left(2 \sigma_{l}^{2}\right)^{\alpha}(\beta(N-l+1) / 2)_{\alpha}
$$


and

$$
|\operatorname{det} X|^{2} \stackrel{\mathrm{d}}{=} \prod_{l=1}^{N} \sigma_{l}^{2} \chi_{\beta l}^{2}
$$

for the beta type I weight

$$
\left\langle|\operatorname{det} X|^{2 \alpha}\right\rangle=\prod_{l=1}^{N} \frac{(\beta(N-l+1) / 2)_{\alpha}}{\left(\beta\left(N+v_{l}\right) / 2\right)_{\alpha}}
$$

and

$$
|\operatorname{det} X|^{2} \stackrel{\mathrm{d}}{=} \prod_{l=1}^{N} \operatorname{Beta}\left[\beta(N-l+1) / 2, \beta\left(v_{l}+l-1\right) / 2\right] ;
$$

for the beta type II weight

$$
\left\langle|\operatorname{det} X|^{2 \alpha}\right\rangle=\prod_{l=1}^{N}(\beta(N-l+1) / 2)_{\alpha}\left(\beta \omega_{l} / 2\right)_{-\alpha}
$$

and

$$
|\operatorname{det} X|^{2} \stackrel{\mathrm{d}}{=} \prod_{l=1}^{N} \operatorname{BetaP}\left[\beta(N-l+1) / 2, \beta \omega_{l} / 2\right]
$$

Proof. In the Gaussian case $f_{j}(u)$ in 2.15 is proportional to $e^{-u / 2 \sigma_{j}^{2}}$. The dependence in the integrand on $r_{l l}$ thus factorises, showing immediately that the joint distribution of $\left\{r_{j j}\right\}$ is proportional to

$$
\prod_{j=1}^{N} r_{j j}^{\beta(N-j+1)-1} e^{-r_{j j}^{2} / 2 \sigma_{j}^{2}} .
$$

Recalling 2.10, the results 2.16 and 2.17) follow as a corollary.

In the case of the beta type I weight, we have that $f_{j}(u)$ is proportional to $(1-u)^{v_{j} / 2-1}, 0<u<1$. The dependence on $r_{j j}$ is determined by the change of variables $r_{l j} \mapsto\left(1-r_{j j}^{2}\right)^{1 / 2} r_{l j}(l<j)$, with $0<r_{j j}<1$ in 2.15). This shows that the joint PDF of $\left\{r_{j j}\right\}$ is proportional to

$$
\prod_{j=1}^{N} r_{j j}^{\beta(N-j+1)-1}\left(1-r_{j j}^{2}\right)^{\beta\left(j-1+v_{j}\right) / 2-1} .
$$

Upon recalling 2.10, we deduce from this both 2.18) and 2.19).

The workings needed to deduce 2.20 and 2.21) for the beta type II weight, proceeds in an analogous way to the beta type I case, except now the change of variables is $r_{l j} \mapsto\left(1+r_{j j}^{2}\right)^{1 / 2} r_{l j}(l<j)$. 
Remark 3. The above working applies simultaneously to cases that the entries of the random matrix are real, complex or quaternion. In the real case $(\beta=1)$ this then provides a proof of Ruben's result Proposition 1 Ruben's proof [31] is seemingly quite different, making use of (amongst other geometrical/ statistical constructions) the exact form of the distribution of an isotropic random vector in $\mathbb{R}^{n}$ projected orthogonally onto a lower dimensional subspace.

The moments for the Gaussian weight for all three number systems, and similarly the moments for the beta type I weight with $v_{l}=0$ (uniform on the sphere) and $\beta v_{l} / 2=1$ (uniform on the ball), have been computed in the recent work [12, Prop. 8]; see also [22] in the Gaussian case. The working relies on (Miles version of) the Blaschke-Petkantschin formula from integral geometry.

Remark 4. As already mentioned below (2.1), a row distribution obtained from the beta type I weight with $v \rightarrow 0^{+}$and $\beta=1$ gives the Gram ensemble in which rows are sampled uniformly from the surface of the unit sphere in $\mathbb{R}^{N}$. As noted in [28], the latter sampling can be done by normalising a standard Gaussian vector, which has the consequence that for $Y$ a member of the Gram ensemble and $X$ a standard Gaussian matrix

$$
\prod_{l=1}^{N} \chi_{\beta N}^{2}|\operatorname{det} Y|^{2} \stackrel{\mathrm{d}}{=}|\operatorname{det} X|^{2} .
$$

The moment formulas 2.16) (with $\sigma_{l}=1 / 2$ ) and 2.18 (with $v_{l}=0$ ) are consistent with this relation.

We remark too that the construction of vectors with distributions 2.1), as given in the paragraph subsequent to 2.1 , extend naturally to the setting of complex and quaternion entries. Thus in the Gaussian case, the independent part of each entry is to be a normal random variable with mean zero and standard deviation $\sigma$. For the beta type I weight with $v \in \mathbb{N}_{0}$, one chooses a standard Gaussian vector with $N+v_{0}$ complex or quaternion entries, normalises it to obtain a vector chosen uniformly from the unit sphere in $\mathbb{C}^{N+v_{0}}$ or $\mathbb{H}^{N+v_{0}}$, and restricts to the first $N$ components; see [9. Eq. (3.113)]. Choosing a $1 \times N$ row vector $\mathbf{v}$ with standard complex or quaternion entries, then forming $(1 / \sqrt{w}) \mathbf{v}$ with $w \stackrel{\mathrm{d}}{=} \chi_{\omega}^{2}$ gives the sought generalisation of the beta type II weight.

Of particular interest, in light of (1.11) with $k=N$, and its generalisation to the complex and quaternion cases, is the expected value of $\log |\operatorname{det} X|$.

Corollary 5. Use $\beta$ to distinguish the number field corresponding to the entries of X. 
Let $\Psi(x):=\frac{d}{d x} \log \Gamma(x)$ denote the digamma function. For the Gaussian weight

$$
\langle\log |\operatorname{det} X|\rangle=\frac{1}{2} \sum_{l=1}^{N}\left(\log 2 \sigma_{l}^{2}+\Psi(\beta(N-l+1) / 2)\right) ;
$$

for the beta type I weight

$$
\langle\log |\operatorname{det} X|\rangle=\frac{1}{2} \sum_{l=1}^{N}\left(\Psi(\beta(N-l+1) / 2)-\Psi\left(\beta\left(N+v_{l}\right) / 2\right)\right) ;
$$

and for the beta type II weight

$$
\langle\log |\operatorname{det} X|\rangle=\frac{1}{2} \sum_{l=1}^{N}\left(\Psi(\beta(N-l+1) / 2)-\Psi\left(\beta \omega_{l} / 2\right)\right) .
$$

Specifically, due to each of the matrix ensembles considered in this section having the invariance (1.7), forming the random product matrix [1.9), we have from (1.11) that the sum of the Lyapunov exponents satisfy

$$
\mu_{1}+\cdots+\mu_{N}=\langle\log |\operatorname{det} X|\rangle
$$

and are thus given explicitly by the results of Corollary 5

\subsection{Noncentral Wishart matrices}

Consider the Gaussian case of (2.1), and suppose $\sigma_{i}=\sigma$ for each row $i$ of the corresponding random matrix $X$. Then the joint distribution of the elements is proportional to $e^{-\operatorname{Tr} X^{T} X / 2 \sigma^{2}}$. The latter exhibits an invariance under the class of transformations $X \mapsto R_{2} X R_{1}$, where $R_{1}, R_{2}$ are real orthogonal matrices, extending the invariance (1.7). Let $c$ be a scalar, and introduce the random matrix $Y:=c I_{N}+X$, where $I_{N}$ denotes the identity. This is invariant under transformations of the form

$$
Y \mapsto R^{T} Y R,
$$

for $R$ real orthogonal. Forming $W=Y^{T} Y$ then gives an example of a noncentral Wishart matrix; see e.g. [24]. According to Constantine [5], generalised to the complex case in [7, Eq. (21)], with $\tilde{c}=c^{2} / 2 \sigma^{2}$ we have

$$
\begin{aligned}
\left\langle(\operatorname{det} W)^{\alpha}\right\rangle & =\prod_{l=1}^{N}\left(2 \sigma^{2}\right)^{\alpha}(\beta(N-l+1) / 2)_{\alpha} e^{-N \tilde{c}}{ }_{1} F_{1}^{(2 / \beta)}\left(\alpha+\beta N / 2 ; \beta N / 2 ; \tilde{c} I_{N}\right) \\
& =\prod_{l=1}^{N}\left(2 \sigma^{2}\right)^{\alpha}(\beta(N-l+1) / 2)_{\alpha} F_{1} F_{1}^{(2 / \beta)}\left(-\alpha, \beta N / 2 ;-\tilde{c} I_{N}\right)
\end{aligned}
$$


where the second equality follows from a generalization of one of Kummer's transformations; see e.g. [24, Th. 7.4.3], [9. Eq. (13.16)]. Here ${ }_{1} F_{1}^{(2 / \beta)}$ is, for $\beta=1,2$ and 4 , a hypergeometric function of matrix argument $X$, dependent on its eigenvalues only (see e.g. [9. Ch. 13]. Since the hypergeometric function is normalised to unity for $X$ equal to the zero matrix, we see that 2.27) correctly reduces to 2.3) in this special case. On the other hand, for $c \neq 0$ the functional dependence on $\alpha$ is now much more complicated.

When $\alpha$ is a positive integer, the series form of ${ }_{1} F_{1}^{(2 / \beta)}$ in the second line of 2.27 terminates (see e.g. [9, eq. (13.2)]), leaving a polynomial in $c$. This is consistent with the LHS of 2.27, as implied by the meaning of $W$. For $\tilde{c}$ large, a known [6. Th. 3.2], [23, \$7] asymptotic formula for ${ }_{1} F_{1}^{(2 / \beta)}$ in the case $\beta=1$, appropriately generalised to all $\beta>0$ using [9. Eqns. (13.12), (13.4), (13.2)] gives

$$
\left\langle\left(\operatorname{det}\left|\mathbb{I}_{N}+\frac{1}{c} X\right|\right)^{2 \alpha}\right\rangle \sim{ }_{2} F_{0}^{(2 / \beta)}\left(1-\beta / 2-\alpha,-\alpha ; \frac{1}{\tilde{c}} \mathbb{I}_{N}\right) .
$$

By differentiating with respect to $\alpha$ and setting $\alpha=0$, this gives that to leading order

$$
\left\langle\log \operatorname{det}\left|\mathbb{I}_{N}+\frac{1}{c} X\right|\right\rangle \underset{c \rightarrow \infty}{\sim} \frac{\sigma^{2} N(\beta / 2-1)}{c^{2}} .
$$

Notice that this vanishes for $\beta=2$. In fact in the case $\beta=2$, differentiating 2.28) with respect to $\alpha$ and setting $\alpha=0$ gives zero for each term in the asymptotic expansion. This indicates that then the large $c$ decay is exponentially fast. In the case $N=1$, the matrix hypergeometric function in 2.27) reduces to the classical confluent hypergeometric function, and the exponentially small term has been exhibited explicitly in [27].

In keeping with 2.25), updated so that the RHS is given by the LHS of 2.29, the result 2.29) gives the leading term in the large $c$ asymptotic expansion for the sum of the Lyapunov exponents of the random product matrix formed by $\left(\mathbb{I}_{N}+\frac{1}{c} X\right)$.

\section{Largest Lyapunov exponent}

\subsection{General formalism}

Consider the three random matrix ensembles specified in terms of the row distributions (2.1). Extend each by allowing for either real, complex or quaternion entries. Now form the random product matrix (1.9). Since each $X_{i}$ is invariant under right multiplication by a unitary matrix with elements from 
the corresponding number field, we know from [4, 25] that the Lyapunov exponents satisfy 1.11 , with $X^{T}$ replaced by $X^{\dagger}$ in the complex and quaternion cases. This is a dramatic simplification from 1.10, or the characterisation from Oseledec's multiplicative ergodic theorem [26], which tells us that the spectrum of $\lim _{m \rightarrow \infty}\left(P_{m}^{\dagger} P_{m}\right)^{1 / 2 m}$ is given by $\left\{e^{\mu_{k}}\right\}_{k=1}^{N}$.

In the case $k=1$, (1.11) involves the sum of the absolute values squared of the first column of $X$ (since the rows of $X$ are isotropic, this may as well be any one of the $N$ columns of $X$ ). Thus

$$
\mu_{1}=\frac{1}{2} \int_{0}^{\infty}(\log t) p(t) d t
$$

where $p(t)$ is the PDF for $\sum_{l=1}^{N}\left|x_{l, 1}\right|^{2}$, with $x_{l, 1}$ denoting the entries in the first column of $X$. To compute $p(t)$, the first task is to deduce from the row distributions (2.1) the marginal distribution of a single element.

Proposition 6. Consider a random vector $\mathbf{x}=\left(x_{1}, \ldots, x_{N}\right)$, with entries that are either real $(\beta=1)$, complex $(\beta=2)$ or quaternion $(\beta=4)$. Let the PDF of the distribution of $\mathbf{x}$ be given by one of the three functional forms in 2.1), appropriately modified in the complex and quaternion cases (recall the paragraph above (2.9)). Each entry $x_{i}$ then involves $\beta$ independent reals. In the Gaussian case, the PDF of the marginal distribution of a single entry $x$ of $\mathbf{x}$ is

$$
\left(2 \pi \sigma^{2}\right)^{-\beta / 2} e^{-|x|^{2} / 2 \sigma^{2}}
$$

for the beta type I weight it is given by

$$
\left.\frac{\Gamma(\alpha+\beta / 2)}{\pi^{\beta / 2} \Gamma(\alpha)}\left(1-|x|^{2}\right)^{\alpha-1}\right|_{\alpha=\beta(N-1+v) / 2^{\prime}} \quad|x|<1 ;
$$

and for the beta type II weight by

$$
\frac{\Gamma((\omega+\beta) / 2)}{\pi^{\beta / 2} \Gamma(\omega / 2)}\left(1+|x|^{2}\right)^{-\frac{1}{2}(\omega+\beta)} .
$$

Proof. In the complex case an element is of the form $x^{(1)}+i x^{(2)}$ with $x^{(1)}, x^{(2)} \in$ $\mathbb{R}$ and $|x|^{2}=\left(x^{(1)}\right)^{2}+\left(x^{(2)}\right)^{2}$. In the quaternion case, written for present purposes in terms of its units $\{1, i, j, k\}$, an element is of the form $x=x^{(1)}+$ $i x^{(2)}+j x^{(3)}+k x^{(4)}$ with $x^{(s)} \in \mathbb{R},(s=1, \ldots, 4)$ and $|x|^{2}=\sum_{s=1}^{4}\left(x^{(s)}\right)^{2}$. By the fact that the PDFs 2.1 are functions only of $|\mathbf{x}|^{2}$, for the given number system each component $x$ has the same distribution. The task to compute this distribution is to integrate the respective PDFs over all components but one. Due to the factorisation property of the Gaussian, the result $(3.2)$ then follows immediately for this weight. 
Consider next the beta type I weight. Suppose for definiteness that the component of interest is $x_{1}$. For each of the other components $\left\{x_{j}\right\}_{j=2, \ldots, N}$ we change variables $x_{j} \mapsto\left(1-\left|x_{1}\right|^{2}\right)^{1 / 2} x_{j}$. This shows that the marginal PDF of $x_{1}$ is proportional to

$$
\left(1-|x|^{2}\right)^{\beta(N-1+v) / 2-1}, \quad|x|<1,
$$

thus implying $3 \cdot 3$.

The analogous change of variables for the beta type II weight gives that the marginal PDF of $x_{1}$ is proportional to

$$
\left(1+|x|^{2}\right)^{-\frac{1}{2}(\omega+\beta)},
$$

thus implying 3.4.

As in the setting of Section 2, we allow for the parameters $\sigma, v, \omega$ in 3.2, (3.3), 3.4 respectively to be different for different rows.

\subsection{The Gaussian case}

The PDF $p(t)$ in 3.1 is then the PDF for $S_{N}=Y_{1}+\cdots+Y_{N}$, where each $Y_{j}$ has distribution Gamma $\left[\beta / 2,1 / 2 \sigma_{j}^{2}\right]$. An infinite series for this PDF, with coefficients defined recursively, is given in [32]. A closed form expression is available in the special case that

$$
1 / 2 \sigma_{1}^{2}=\cdots=1 / 2 \sigma_{N_{0}}^{2}=b_{1}, \quad 1 / 2 \sigma_{N_{0}+1}^{2}=\cdots=1 / 2 \sigma_{N}^{2}=b_{2},
$$

since then $S_{N} \stackrel{\mathrm{d}}{=} X_{1}+X_{2}$, where $X_{1}, X_{2}$ have distribution Gamma $\left[\beta N_{0} / 2, b_{1}\right]$, Gamma $\left[\beta\left(N-N_{0}\right) / 2, b_{2}\right]$ respectively. From this characterisation, it is straightforward to derive (see e.g. [3]) that

$$
p(t)=\frac{b_{1}^{\beta N_{0} / 2} b_{2}^{\beta\left(N-N_{0}\right) / 2}}{\Gamma(\beta N / 2)} t^{\beta N / 2-1} e^{-b_{2} t}{ }_{1} F_{1}\left(\beta N_{0} / 2, \beta N / 2 ;\left(b_{2}-b_{1}\right) t\right) .
$$

Use of computer algebra gives

$$
\int_{0}^{\infty} t^{s} p(t) d t=\frac{\Gamma(\beta N / 2+s)}{\Gamma(\beta N / 2)} b_{2}^{-s}{ }_{2} F_{1}\left(\beta N_{0} / 2,-s ; \beta N / 2 ; 1-\frac{b_{2}}{b_{1}}\right) .
$$

In the case $N_{0}=0$, this reclaims (2.3). Also, the limit $b_{1} \rightarrow \infty$ reclaims 2.3 but with $N$ replaced by $N-N_{0}$. This latter property is consistent with the effect of taking $b_{1} \rightarrow \infty$ being to set the first $N_{0}$ rows equal to zero. Taking the derivative of 3.8 with respect to $s$ and setting $s=0$ gives the evaluation of $\mu_{1}$ according to 3.1. 
Proposition 7. Consider a product of independent Gaussian random matrices 2.2., with variances given by (3.6), and rows ordered so that $b_{2} \leq b_{1}$. We have

$$
\begin{aligned}
\mu_{1}=\frac{1}{2}( & \Psi(\beta N / 2)-\log b_{2}+\frac{\Gamma(\beta N / 2)}{\Gamma\left(\beta N_{0} / 2\right) \Gamma\left(\beta\left(N-N_{0}\right) / 2\right)} \\
& \left.\times \int_{0}^{1} x^{\beta N_{0} / 2-1}(1-x)^{\beta\left(N-N_{0}\right) / 2-1} \log \left(1-\left(1-\frac{b_{2}}{b_{1}}\right) x\right) d x\right) .
\end{aligned}
$$

Proof. This follows from the stated strategy, upon use of the Euler integral form of ${ }_{2} F_{1}$.

The integral formula 3.9 is well suited to an asymptotic analysis.

Corollary 8. Consider (3.9). For $N \rightarrow \infty$ with $N_{0} / N$ fixed, $b_{2} / b_{1}$ fixed, we have

$$
\mu_{1} \sim \frac{1}{2}\left(\Psi(\beta N / 2)-\log b_{2}+\log \left(1-\left(1-\frac{b_{2}}{b_{1}}\right) \frac{N_{0}}{N}\right)\right) .
$$

If in addition $b_{1}, b_{2}$ are proportional to $N$, this further simplifies to give

$$
\mu_{1} \sim \frac{1}{2} \log \left(\frac{\beta}{2}\left(\frac{N-N_{0}}{b_{2}}+\frac{N_{0}}{b_{1}}\right)\right) .
$$

In the case that $N_{0}=N-1, b_{1}$ is proportional to $N$ and $b_{2}$ is fixed, we have

$$
\mu_{1} \sim \frac{1}{2}\left(\log \frac{\beta N}{2 b_{1}}+\frac{(\beta / 2)^{\beta / 2}}{\Gamma(\beta / 2)} \int_{0}^{\infty} e^{-\beta x / 2} x^{\beta / 2-1} \log \left(1+\frac{b_{1}}{N b_{2}} x\right) d x\right) .
$$

Proof. In the setting of 3.10 , the integrand in 3.9 has it maximum value at $x=N_{0} / N$. Since the ratio of gamma functions prefactor is just the reciprocal of the same integral without the log term, 3.10 follows. In the latter, using the fact that for large $x, \Psi(x) \sim x+\mathrm{O}(1 / x)$, gives 3.11. To obtain 3.12, we first change variables $x \mapsto 1-x$ in 3.9 . Manipulation of the log term in the integrand shows

$$
\begin{aligned}
\mu_{1}=\frac{1}{2}(\Psi( & \beta N / 2)-\log b_{1}+\frac{\Gamma(\beta N / 2)}{\Gamma\left(\beta N_{0} / 2\right) \Gamma\left(\beta\left(N-N_{0}\right) / 2\right)} \\
& \left.\times \int_{0}^{1} x^{\beta\left(N-N_{0}\right) / 2-1}(1-x)^{\beta\left(N_{0} / 2-1\right.} \log \left(1-\left(1-\frac{b_{1}}{b_{2}}\right) x\right) d x\right) .
\end{aligned}
$$

Again, the ratio of gamma function prefactor can be written as the same integral without the $\log$ term. Rewriting as such, and setting $N_{0}=1$, the asymptotic form follows by changing variables $x \mapsto x / N$, and taking $N$ large. 
Remark 9. The matrix $\Sigma$ in 2.2 , in the setting of 3.6 , has for the sum of its diagonal entries

$$
\operatorname{Tr} \Sigma=\frac{N-N_{0}}{2 b_{2}}+\frac{N_{0}}{2 b_{1}} .
$$

Thus 3.11 can be rewritten

$$
\mu_{1} \sim \frac{1}{2} \log (\beta \operatorname{Tr} \Sigma)
$$

This has been derived previously in [17] (the additional factor of $\beta$ in the logarithm on the LHS of 3.13 is due to our use of standard real Gaussians, whereas in [17], standard complex (quaternion) Gaussians are used for $\beta=2$ $(\beta=4))$. With $S_{N}$ defined above 3.6 , another viewpoint on 3.13 is that to leading order for large $N$,

$$
\left\langle\log S_{N}\right\rangle \sim \log \left\langle S_{N}\right\rangle
$$

For $\beta=2$ an evaluation easily seen to be equivalent to 3.12 with $\beta=2$ is also given in [17, Eq. (2)]. In addition, the latter reference also treats the case $\beta=1$, where $2 b_{1} / N=1,2 b_{2}=t$, and we read off from Thm. 1.3 therein the formula

$$
\lim _{N \rightarrow \infty} \mu_{1}=\frac{1}{2} e^{t / 2} \int_{1}^{\infty} e^{-t x / 2} \frac{d x}{\sqrt{x}(\sqrt{x}+1)} .
$$

Numerical evaluation indicates that our formula is equivalent to this, although we don't have a direct proof.

The evaluation (3.9) of the largest Lyapunov exponent for products of independent Gaussian random matrices (2.2), with variances given by 3.6 , is based on the explicit evaluation (3.7) of the PDF $p(t)$ in (3.1). It is an elementary fact that in the general variance case $\hat{p}(k)$, the Fourier transform of $p(t)$, can be evaluated explicitly. From this starting point, and with the use of complex analysis, Kargin [17] has obtained an integral evaluation of the largest Lyapunov exponent in the case of general variances $1 / 2 \sigma_{i}^{2}=b_{i}$,

$$
\begin{aligned}
2 \mu_{1} & =-\gamma+\log 2+\int_{0}^{\infty}\left(\chi_{x \in(0,1)}-\prod_{l=1}^{N}\left(1+\frac{x}{2 b_{l}}\right)^{-\beta / 2}\right) \frac{d x}{x} \\
& =-\gamma+\int_{0}^{\infty}\left(\chi_{x \in(0,1)}-\prod_{l=1}^{N}\left(1+\frac{x}{b_{l}}\right)^{-\beta / 2}\right) \frac{d x}{x}
\end{aligned}
$$

where $\gamma$ denotes Euler's constant and $\chi_{J}=1$ for $J$ true, $\chi_{J}=0$ otherwise (we have added $\log \beta$ to [17, Eq. (1)] due to our use of standard real Gaussians; recall the discussion below (3.13). Note that in the special case $3.6,3.15$ 
remains distinct from 3.9 . Here we provide a derivation of 3.15 that is independent of complex analysis, and thus distinct from that in [17].

Proof of 3.15. In [36], the exact formula 4.29 below was given for the largest Lyapunov exponent in the case of the product of independent matrices of the form $\mathbb{I}_{2}+\frac{1}{c} G$, where $G$ is a $2 \times 2$ standard Gaussian matrix. This calculation was based on the particular integral form of the logarithm function

$$
\log y=\int_{0}^{\infty} \frac{e^{-t}-e^{-t y}}{t} d t, \quad y>0 .
$$

Applying 3.16 to the present setting of real, complex or quaternion standard Gaussian entries along each row, with all independent parts distributed as $\mathrm{N}\left[0, \sqrt{1 /\left(2 b_{l}\right)}\right]$ (i.e. a mean zero normal with standard deviation $\sqrt{1 /\left(2 b_{l}\right)}$ ), shows

$$
2 \mu_{1}=\int_{0}^{\infty}\left(e^{-t}-\prod_{l=1}^{N} \prod_{s=1}^{\beta}\left\langle e^{-t\left(x_{l}^{(s)}\right)^{2}}\right\rangle_{x_{l}^{(s)} \in \mathrm{N}\left[0, \sqrt{1 /\left(2 b_{l}\right)}\right]}\right) \frac{d t}{t} .
$$

Computing the average is elementary, reducing 3.17 to

$$
2 \mu_{1}=\int_{0}^{\infty}\left(e^{-t}-\prod_{l=1}^{N}\left(1+\frac{t}{b_{l}}\right)^{-\beta / 2}\right) \frac{d t}{t} .
$$

Upon minor manipulation, this reclaims 3.15).

\subsection{Beta type I distribution}

Here we consider the case that each row of the random matrix has beta type I distribution 3.3 with $v \mapsto v_{l}$, appropriately generalised to allow for complex or quaternion entries. An evaluation of the Lyapunov exponent as an infinite series can be given.

Proposition 10. In the above specified setting,

$$
\mu_{1}=\frac{1}{2} \sum_{n=-\infty}^{\infty} c_{n} \int_{0}^{\beta N}(\log x) e^{2 \pi i n x / \beta N} d x
$$

where

$$
c_{n}=\frac{1}{\beta N} \prod_{l=1}^{N}{ }_{1} F_{1}\left(\beta / 2, \beta / 2+\alpha_{l} ;-2 \pi i n / \beta N\right),
$$

with $\alpha_{l}=\beta\left(N+v_{l}-1\right) / 2$.

Proof. Generally, in the setting that each $x_{l}$ has PDF $q_{l}(x)$, the Fourier transform $\hat{p}(k)$ of the PDF for the distribution of $S_{N}:=\sum_{l=1}^{N}\left|x_{l}\right|^{2}$ is, according to the 
convolution formula, given by

$$
\hat{p}(k)=\prod_{l=1}^{N} \int_{\mathbb{R}^{\beta}} e^{i k|x|^{2}} q_{l}(x) d x^{(1)} \cdots d x^{(\beta)} .
$$

For $q_{l}(x)$ equal to 3.3 with $v \mapsto v_{l}, q_{l}(x)$ is supported on $|x|<1$ and so $p(x)$ is supported on $(0, \beta N)$. Thus for $x \in(0, \beta N)$ we have the Fourier series

$$
p(x)=\sum_{n=-\infty}^{\infty} c_{n} e^{2 \pi i n x / \beta N}, \quad c_{n}=\frac{1}{\beta N} \int_{0}^{\beta N} p(x) e^{-2 \pi i x n / \beta N} d x .
$$

Making use of (3.21) it follows that

$$
c_{n}=\frac{1}{\beta N} \prod_{l=1}^{N} \int_{|x|<1} e^{-2 \pi i|x|^{2} n / \beta N} q_{l}(x) d x^{(1)} \cdots d x^{(\beta)} .
$$

Substituting (3.3) for $q_{l}(x)$, and changing variables to polar coordinates, an integral representation of the confluent hypergeometric function results, and shows the integral can be evaluated to give 3.20. Recalling 3.1, 3.19) follows.

Use of the integral form of the logarithm (3.16) allows for an alternative expression for the Lyapunov exponent, which is the analogue of 3.18).

Proposition 11. An alternative expression to the result of Proposition 10 is the formula

$$
2 \mu_{1}=\int_{0}^{\infty}\left(e^{-t}-\prod_{l=1}^{N}{ }_{1} F_{1}\left(\beta / 2, \beta / 2+\alpha_{l} ;-t\right)\right) \frac{d t}{t} .
$$

Proof. The essential point is that for $p(x)$ given by 3.3 ,

$$
\left\langle e^{-t|x|^{2}}\right\rangle_{x \in p(x)}=\int_{|x|<1} e^{-t|x|^{2}} p(x) d x^{(1)} \cdots d x^{(\beta)}={ }_{1} F_{1}\left(\beta / 2, \beta / 2+\alpha_{l} ;-t\right) .
$$

This follows from the use of polar coordinates, and recognition of the resulting expression as a standard integral form of ${ }_{1} F_{1}$.

We note from 3.5 with $\beta=1$ that the choices $(N, v)=(2,1)$, and $(N, v)=$ $(3,0)$, give the marginal distribution of each entry as uniform on $(-1,1)$. Furthermore, entries in different rows are independent, so the PDF $p(t)$ in 3.1 is then the PDF for $\sum_{i=1}^{N} U_{i}^{2}$, where each $U_{i}$ is an independent random variable uniformly distributed on $(0,1)$. For general $N$, this random sum has been the topic of the recent works [34, 13], and for $N=2$ arose in the recent study [15]. 


\subsection{Beta type II distribution}

As for the Gaussian and beta type I cases, the identity (3.16) again quickly leads to an explicit integral formula for the largest Lyapunov exponent in the case of beta type II distribution.

Proposition 12. Specify that each row of the random matrix has beta type II distribution (3.4) with $\omega \mapsto \omega_{l}$, appropriately generalised to allow for complex or quaternion entries. The corresponding Lyapunov exponent is given by

$$
2 \mu_{1}=\int_{0}^{\infty}\left(e^{-t}-\prod_{l=1}^{N}\left(\frac{\Gamma\left(\omega_{l}+\beta / 2\right)}{\Gamma\left(\omega_{l} / 2\right)} U\left(\beta / 2,1-\omega_{l} / 2 ; t\right)\right) \frac{d t}{t}\right.
$$

where $U(a, b ; x)$ denotes the confluent hypergeometric function of the second kind.

Proof. Analogous to 3.23, for $p(x)$ given by 3.4,

$$
\left\langle e^{-t|x|^{2}}\right\rangle_{x \in p(x)}=\int_{\mathbb{R}^{\beta}} e^{-t|x|^{2}} p(x) d x^{(1)} \cdots d x^{(\beta)}=\frac{\Gamma\left(\omega_{l}+\beta / 2\right)}{\Gamma(\omega / 2)} U\left(\beta / 2,1-\omega_{l} / 2 ; t\right),
$$

where again polar coordinates are used, and a standard integral form of $U(a, b ; x)$ is recognised to obtain the final equality.

Remark 13. The structural similarity between 3.24, 3.22 and 3.15 is evident. In fact the latter can be reclaimed from either of 3.24 or 3.22. Consider for definiteness the former. Set $\omega_{l}=2 b_{l} L$. From the integral form of $U(a, b ; x)$ as implicit in 3.25, we see that as $L \rightarrow \infty$

$$
\frac{\Gamma\left(\omega_{l}+\beta / 2\right)}{\Gamma\left(\omega_{l} / 2\right)} U\left(\beta / 2,1-\omega_{l} / 2 ; x\right) \rightarrow \frac{1}{\left(1+x / b_{l}\right)^{\beta / 2}}
$$

and thus $2 \mu_{1}+\log L$ tends to 3.15.

Remark 14. There is an analogue of the large $N$ result 3.13 in both the type I and type II beta cases,

$$
\mu_{1} \sim \frac{1}{2} \log \sum_{j=1}^{N}\left(1+v_{j}\right)^{-1}, \quad \mu_{1} \sim \frac{1}{2} \log \sum_{j=1}^{N}\left(\omega_{j}-2 / \beta\right)^{-1},
$$

respectively. This is a consequence of 3.14.

Remark 15. As a part of Remark 4 methods to sample an element in the first column of $X, x_{l, 1}$ say, in each of the 3 cases under consideration - rows of $X$ sampled according to the PDFs and their extension to their extension to the 
complex and quaterion cases - was given. This then allows for a Monte Carlo approximation to 3.1. according to

$$
\mu_{1} \approx \frac{1}{2 M} \sum_{j=1}^{M} \log \left(\sum_{l=1}^{N}\left|x_{l, 1}^{(j)}\right|^{2}\right),
$$

where $x_{l, 1}^{(j)}$ denotes the $j$-th sample of $x_{l, 1}$ and $M$ is the total number of times the column is sampled. This allows for (3.18), 3.22) and (3.24) to be checked numerically; agreement is found although the integrand in 3.22 typically requires high precision evaluation to obtain numerical stability.

\section{Noncentral Wishart matrices}

In $\$ 2.2$ the random matrix $Y=c I_{N}+X$, where $X$ has i.i.d. real Gaussian entries $\mathrm{N}[0, \sigma]$, was introduced, as were the complex and quaternion analogues. It has been observed in [36] that the invariance 2.26 implies the simple formula (1.11) - with the matrix $X$ replaced by $Y$ - for the sum of the Lyapunov exponents. Thus in the case $k=N$, for $c$ large the asymptotic formula 2.29) holds true. A similar strategy allows for the derivation of the corresponding result in the general $k$ case.

Let $Y_{k}$ denote first $k$ columns of the matrix $Y$. The matrix $W_{k}:=Y_{k}^{+} Y_{k}$ is, as for the case $k=N$, an example of a non-central Wishart matrix. From the work of Constantine [5], generalised to the complex case in [7, Eq. (21)], with $\tilde{c}=c^{2} / 2 \sigma^{2}$ we have

$$
\begin{aligned}
\left\langle\left(\operatorname{det} W_{k}\right)^{\alpha}\right\rangle & =\prod_{l=1}^{k}\left(2 \sigma^{2}\right)^{\alpha}(\beta(N-l+1) / 2)_{\alpha} e^{-k \tilde{c}}{ }_{1} F_{1}^{(2 / \beta)}\left(\alpha+\beta N / 2 ; \beta N / 2 ; \tilde{c} I_{k}\right) \\
& =\prod_{l=1}^{k}\left(2 \sigma^{2}\right)^{\alpha}(\beta(N-l+1) / 2)_{\alpha}{ }_{1} F_{1}^{(2 / \beta)}\left(-\alpha, \beta N / 2 ;-\tilde{c} I_{k}\right) .
\end{aligned}
$$

More revealing is the simplification of 4.26) obtained upon substitution of the large $\tilde{c}$ asymptotic expansion [6, Th. 3.2], [23, \$7] for ${ }_{1} F_{1}^{(2 / \beta)}$ in the case $\beta=1$ known from [6. Th. 3.2], [23, \$7], appropriately generalised to all $\beta>0$ as noted above 2.28. This gives as a generalisation of 2.28

$$
\left\langle\left(\operatorname{det} W_{k}\right)^{\alpha}\right\rangle \sim{ }_{2} F_{0}^{(2 / \beta)}\left(1-\alpha-\beta(N-k+1) / 2,-\alpha ; \frac{1}{\tilde{c}} \mathbb{I}_{k}\right) .
$$

Differentiating with respect to $\alpha$ and setting $\alpha=0$, it then follows that as a generalisation of 2.29), to leading order

$$
\mu_{1}+\cdots+\mu_{k}=\frac{1}{2}\left\langle\log \operatorname{det} W_{k}\right\rangle \underset{c \rightarrow \infty}{\sim} \frac{\sigma^{2} k(\beta(N-k+1) / 2-1)}{c^{2}} .
$$


Notice that in the case $k=N-1, \beta=1$ the coefficient on the RHS vanishes. Moreover 4.27) shows that each term in the asymptotic expansion vanishes, indicating that the large $c$ decay is in fact exponentially fast. In the particular case of this type, $\beta=1, N=2, k=1$ it was shown in [36. Eq. (72)] that

$$
\mu_{1}=\frac{1}{2} \int_{c^{2} / 2 \sigma^{2}}^{\infty} \frac{e^{-u}}{u} d u,
$$

which decays as a Gaussian at infinity.

Setting $c=0$ in (4.26) shows that for $X$ a Gaussian matrix with real, complex or quaternion entries, with parts of each entry distributed as $\mathrm{N}[0, \sigma]$,

$$
\left\langle\operatorname{det}\left(X_{N \times k}^{\dagger} X_{N \times k}\right)\right\rangle=\prod_{l=1}^{k}\left(2 \sigma^{2}\right)^{\alpha}(\beta(N-l+1) / 2)_{\alpha} .
$$

This allows the RHS of (1.11) to be computed, telling us that

$$
\mu_{1}+\cdots+\mu_{k}=\frac{1}{2}\left(\log \left(2 \sigma^{2}\right)+\sum_{l=1}^{k} \Psi(\beta(N-l+1) / 2)\right)
$$

(cf. [2.22)), as was known previously [25], [10]. Generalisations relating to quantifying the convergence of this Lyapunov spectrum as a Gaussian random variable with particular variances are given in [1, 16, 11]

For $k=1$ and general $N, \beta$, use can be made of 3.1 to get a formula for $\mu_{1}$ which is distinct from that implied by 4.27 . In the present setting the function $p(t)$ in 3.1 is the PDF for $S_{N}=(\sigma / c)^{2} \sum_{j=1}^{N} \sum_{s=1}^{\beta}\left(x_{1 j}^{(s)}\right)^{2}$, where $x_{11}^{(1)}$, is distributed as $\mathrm{N}[c / \sigma, 1]$ and $x_{1 j}^{(s)}, s=1, \ldots, \beta, j=1, \ldots, N,(s, j) \neq$ $(1,1)$ is distributed as $\mathrm{N}[0,1]$. After scaling out the factor of $(\sigma / c)^{2}$, this sum specifies the noncentral $\chi$ squared distribution with $\beta N$ degrees of freedom and noncentrality parameter $\lambda=(c / \sigma)^{2}$. From the known PDF for the latter (see e.g. [35]), we thus have

$$
\mu_{1}=\frac{1}{2} \int_{0}^{\infty} \log (t / \lambda) f(t ; \beta N, \lambda) d t,
$$

where

$$
f(t ; k, \lambda)=\frac{1}{2} e^{-(t+\lambda) / 2}\left(\frac{t}{\lambda}\right)^{k / 4-1 / 2} I_{k / 2-1}(\sqrt{\lambda t}) .
$$

For large $\lambda$, use of the leading asymptotic expansion $I_{v}(z) \sim e^{z} /(2 \pi z)^{1 / 2}$, and expansion of the integrand about its maximum at $t=\lambda$ reclaims 4.28 in the case $k=1$. The form of 4.30 with $\beta=1, N=2$ is different to 4.29 . Both are simple to compute numerically and give the same value. 


\section{Acknowledgements}

This research project is part of the program of study supported by the ARC Centre of Excellence for Mathematical \& Statistical Frontiers, and the Australian Research Council Discovery Project grant DP170102028. The work of JZ was supported by a University of Melbourne Research Scholarship.

\section{References}

[1] G. Akemann, Z. Burda, and M. Kieburg, Universal distribution of Lyapunov exponents for products of Ginibre matrices, J. Phys. A 47 (2014), 395202.

[2] M.S. Bartlett, The vector representation of a sample, Math. Proc. Cambridge Philos. Soc. 30 (1934), 327-340.

[3] J. Bausch, On the efficient calculation of a linear combination of chi-squared random variables with an application in counting string vacua, J. Phys. A 46 (2013), 505202.

[4] J.E. Cohen and C.M. Newman, The stability of large random matrices and their products, The Annals of Prob. 12 (1984), 283-310.

[5] A.G. Constantine, Some noncentral distribution problems in multivariate analysis, Ann. Math. Statist. 34 (1963), 1270-1285.

[6] A.G. Constantine and R.J. Muirhead, Asymptotic expansions for distributions of latent roots in multivariate analysis, J. Mult. Analysis 6 (1976) , 369-391.

[7] X. Cui and Q.T. Zhang, Generic procedure for tightly bounding the capacity of MIMO correlated Rician fading channels, IEEE Trans. Commun. 53 (2005), $890-898$.

[8] J.A. Díaz-García and R. Gutiérrez-Jáimez, Random matrix theory and multivariate statistics, arXiv:0907.1064.

[9] P.J. Forrester, Log-gases and random matrices, Princeton University Press, Princeton, NJ, 2010.

[10] P.J. Forrester, Lyapunov exponents for products of complex Gaussian random matrices, J. Stat. Phys. 151 (2013) , 796-808.

[11] P.J. Forrester, Asymptotics of finite system Lyapunov exponents for some random matrix ensembles, J. Phys. A 48 (2015), 215205. 
[12] P.J. Forrester, Matrix polar decomposition and generalisations of the BlaschkePetkantschin formula in integral geometry, arXiv:1701.04505

[13] P.J. Forrester, Comment on "Sum of squares of uniform random variables" by I. Weissman, arXiv:1804.07861

[14] P.J. Forrester and J.R. Ipsen, Selberg integral theory and Muttalib-Borodin ensembles, Adv. Appl. Math. 95 (2018), 152-176.

[15] P.J. Forrester and J. Zhang, Volumes and distributions for random unimodular complex and quaternion lattices, J. Number Th. (2018) DOI:10.1016/j.jnt.2018.03.010

[16] J.R. Ipsen, Lyapunov exponents for products of rectangular real, complex and quaternionic Ginibre matrices, J. Phys. A 48 (2015), 155204.

[17] V. Kargin, On the largest Lyapunov exponent for products of Gaussian matrices, J. Stat. Phys. 157 (2014), 70-83.

[18] Z. Kabluchko, D. Temesvari and C. Thäle, Expected intrinsic volumes and facet number of random beta-polytopes, arXiv:1707.02253

[19] Z. Kabluchko, A. Marynych, D. Temesvari and C. Thäle, Cones generated by random points on half-spheres and convex hulls of Poisson point processes, arXiv:1801.08008

[20] A.M. Mathai, Random p-content of a p-parallelotope in Euclidean n-space, Adv. Appl. Probab. 31 (1999), 343-354.

[21] A.M. Mathai, An introduction to geometric probability, Gordon and Breach Science Publishers, Amsterdam, 1999.

[22] S.R. Moghadasi, Polar decomposition of the $k$-fold Lebesgue measure on $\mathbb{R}^{n}$, Bull. Aust. Math. Soc. 85 (2012), 315-324.

[23] R.J. Muirhead, Latent roots and matrix variates: a review of some asymptotic results, Ann. Statistics 6 (1978), 5-33.

[24] R. J. Muirhead, Aspects of multivariate statistical theory, Wiley, New York, 1982.

[25] C.M. Newman, The distribution of Lyapunov exponents: exact results for random matrices, Commun. Math. Phys. 103 (1986), 121-126. 
[26] V.I. Oseledec, A multiplicative ergodic theorem. Lyapunov characteristic numbers for dynamical systems, Trans. Moscow Math. Soc. 19 (1968), 197-231.

[27] R.B. Paris, Exponentially small expansions of the confluent hypergeometric function, Appl. Math. Sciences 7 (2013), 6601-6609.

[28] A. Prékopa, On random determinants I, Studia Sci. Mat. Hungar. 2 (1967), 125-132.

[29] M.S. Raghunathan, A proof of Oseledec's multiplicative ergodic theorem, Israel J. Math. 32 (1979), 356-362.

[30] A. Rouault, Asymptotic behavior of random determinants in the Laguerre, Gram and Jacobi ensembles, Alea 3 (2007), 181-230.

[31] H. Ruben, The volume of an isotropic random parallelotope, J, Appl. Probab. 16 (1979), 84-94.

[32] C.H. Sim, Point processes with correlated gamma inter arrival times, Stat. Prob. Letters 15 (1992), 135-141.

[33] Y. Tsaig and D.L. Donoho, Breakdown of equivalence between the minimal $L_{1^{-}}$ norm solution and the sparsest solution, Signal Processing 86 (2006), 533-548.

[34] I. Weissman, Sum of squares of uniform random variables, Statist. Probab. Lett. 129 (2017), 147-154

[35] Wikipedia, Noncentral chi-squared distribution, https://en.wikipedia.org/wiki/Noncentral_chi-squared_distribution

[36] N. Zanon and B. Derrida, Weak disorder expansion of Liapunov exponents in a degenerate case, J. Stat. Phys. 50 (1988), 509-528. 